Research Article

Open Access

\title{
Building Capacity for Pediatric HIV Care in Haiti: Evaluating a Human Resources Training Program
}

\author{
Sarah Elizabeth Davey \\ Department of Pediatric, Brown University, Providence, RI 02912, USA
}

\section{Article Info}

*Corresponding author:
Sarah Elizabeth Davey
Department of Pediatric
Brown University, Providence, RI 02912
USA
E-mail: sarah_davey@brown.edu

Received: April 2, 2018

Accepted: April 9, 2018

Published: May 16, 2018

Citation: Davey SE. Building Capacity for Pediatric HIV Care in Haiti: Evaluating a Human Resources Training Program. Madridge J AIDS. 2018; 2(1): 29-34.

doi: $10.18689 / \mathrm{mja}-1000105$

Copyright: (c) 2018 The Author(s). This work is licensed under a Creative Commons Attribution 4.0 International License, which permits unrestricted use, distribution, and reproduction in any medium, provided the original work is properly cited.

Published by Madridge Publishers

\section{Background}

Haiti is a severely resource-constrained country in the America's and $207^{\text {th }}$ in the world in GDP per capita. The World Bank has estimated that about $68 \%$ of the Haitian population is affected by poverty and about $47 \%$ is affected by extreme poverty $[1,2]$ With such high levels of poverty, Haiti also experiences some of the highest rates of HIV in the world, largely attributable to lack of HIV-specialized care providers throughout the very rural country. In 2013 , WHO and UNAIDS rolled out the " 3 by 5 " initiative, with the target of reaching 3 million people living with HIV/AIDS in low- and middle-income countries by 2005. Regardless of an improvement in access to antiretroviral therapy (ART) care over the past decade, gaps remain in receipt of care. Less than $5 \%$ of the recipients of this care were children, with delivery impeded by barriers such as poor pediatric drug formulations, lack of pediatric diagnostic methods, and, most importantly, a shortage of pediatric care providers [1]. As of 2012, about 150,000 people living in Haiti were HIV-positive, with 12,000 of these being children under the age of 14 years old. Most children are infected through maternal to child transmission, with vertical transmission reported as being between 27-37 [1]. HIV prevalence among pregnant women averages $3.1 \%$ in 2003 and ranges between $0.8 \%$ in the country's western, rural parts, to upwards off $11.8 \%$ in urban areas [1].

There are many contributing factors to the gap between the children in need and those receiving ART, which include constraints in the health care system and human resources, difficulty of perinatal diagnosis (and prevention of maternal to child transmission, PMTCT), early childhood diagnosis, complexity of pediatric ART formulations, lack of funding, and family/social challenges [2]. Testing and treating earlier in the trajectory of infection can lead to a much better prognosis and improvement in quality of life [3]. Early and consistent delivery of ART therapy is cost effective because patients are identified and treated before presenting with costly work-ups and treatments for opportunistic infections. However, with risk factors such as the 2010 earthquake, migration, poverty, disease stigma, and a resource-limited health care system, this makes Haiti's situation even more complex. With a physician density of only 0.25 per 1,000 population: illustrating a critical need for capacity building programs that focus on human resources [3]. In 2006, Haiti adopted national HIV treatment guidelines, but the problem of lack of human resource capacity remains.

St. Damien Hospital for children (SDH) has the largest medical training program for pediatric HIV care in Haiti and has the potential for making a significant impact on the number of HIVspecialized physicians throughout the country [1]. In 2007, SDH began routine HIV testing of all patients (both in- and outpatients). The HIV Training Program for Physicians at St. Damien Hospital (HIVTP) was established in December 2008, with hospital funding and additional support from PEPFAR [4]. The mission of the program is to support children at risk for HIV and to assist in the expansion of support throughout the country by training providers in HIV/AIDS. As of May 2012, a total of 22 providers (with 14 more trained in 2013) had been 
trained: 5 pediatricians and 17 general practitioners. The HIVTP trainers are comprised of 5 pediatricians, 1 general physician, 1 psychologist, 2 social workers, and 1 pharmacist. The program operates as a training course that combines theoretical and practical sessions in both the outpatient HIV clinic, as well as admitted patients with HIV at SDH. Studies performed in SubSaharan Africa on decentralization of pediatric HIV care have argued that in order to reduce the gap between need and delivery of care for children, there needs to be a geographical expansion of services from large urban health care facilities to smaller, rural health facilities where most of the primary care for children in low-resource settings is provided [5]. More pediatric HIV care providers available in low-resource settings will not only improve testing and diagnosis, but also create better drug adherence and retention of care through more readily available clinicians [6]. The SDH HIV clinic is a premiere treatment center with over 400 children on ART (representing $25 \%$ of total children on ART in Haiti). In addition, the HIVTP is narrowing the gap between need and delivery of pediatric HIV care by creating a larger base of HIV-specialized trained clinicians that span across a wider geographical setting [7].

\section{Objective and Specific Aims}

The proposed research will evaluate the multiple facets of an HIV training program in a resource-limited setting. Specifically we hope to evaluate the training curriculum, trainee performance, and impact of Pediatric HIV care in Haiti. This evaluation will provide key information for understanding feasibility of in-country scale-up.

Specific Aim 1: Evaluation of the program curriculum with respect to topic content, program length, practical skills, and lecture modalities.

Approach Internet search of available teaching modalities. Hold focus groups with key informants: program leaders, program graduates, social workers, psychologists, Brown HIV leaders, and other program stakeholders (See Appendix C). Structured survey of trainees and trainers (See Appendix A)

Specific Aim $\mathbf{2}$ Investigate trainee performance as a marker for continued engagement in HIV care (pre-post tests) - (See Appendix B)

Approach: Using raw scores (pre- and post-tests) and performance improvement to develop measures of effect (chi square test) between individual program performance (high, average, and low performance) and level of HIV care (none, few, many) following program completion.

Performance will be measured from pre- and post- test program scores and level of care determination will be retrieved from participant survey.

Specific Aim 3 Measure the clinical impact of the training program on children living with HIV in Haiti.

Approach: Descriptive analysis of graduated trainee's current activity, including percent providing HIV care, percent of patients with HIV in their clinic, and what percent of HIV patients seen in their clinic is on ARV. Data will be collected from structured survey.
Specific Aim 4 Identification of programmatic strengths and weaknesses as it pertains to commonly encountered barriers of providing HIV care in the primary-care setting.

Approach Use results from structured survey. Hold an additional focus group with key informants: program leaders, program graduates, social workers, psychologists, and other program stakeholders.

\section{Participant Population}

The target population for this study are the graduates of the Saint Damien HIVTP, as well as key informants in the development program such as trainers, key clinic staff, and supervisors. The survey will be administered to the group of participants that are publicly known, through the list of the certificate program. Inclusion in the study population for the structured survey includes completion of the Saint Damien HIVTP. Exclusion from the study population for the structured survey includes any individuals who did not meet all of the completion requirements of the training program. Inclusion in the focus group includes being a key informant in the HIVTP; this includes program organizers, directors, lecturers, key clinic staff, and trainers (most of whom have already agreed to participate as part of our working collaborations that exist between Brown University and Saint Damien). All graduates of the program will be contacted first through e-mail (which is kept on file in HIVTP records and we have access to), then by the most up-to-date phone number kept on record if e-mail is unsuccessful. Three attempts will be made at contact via e-mail and ten attempts will be made through phone call before participants are considered not contactable. When subjects are contacted by phone, they will be asked for a working email for survey distribution to keep responses anonymous.

Participants in the HIVTP will be identified through the recorded list provided by program coordinator Dr. Marie Lina Excellent, which includes names and contact information. Aggregate data from both the pre- and post-program evaluations have already been made available to us through Dr. Excellent. Confidentiality in all test scores and identifiable participant information will be ensured through a system of coding. We will use a system of coding by trading all identifiers (name, contact information, etc.) with unique participant ID numbers. We will gather all of the, including linking individual pre-and posttest scores, which will be completely available at the time of completing the research and we will not keep any identifiers with data (only unique ID numbers). All information will be collected in the HIV training program headquarters, under the HIVTP program director's supervision, and all information that is reviewed will not leave the program headquarters unless under a coded identification number.

\section{Methods and Data Collection Procedures}

Our methodologies will be three-fold with surveys, focus groups, and collection of program statistics. In June 2014, we propose to travel to SDH in Tabarre, Haiti to conduct a program follow-up and evaluation of HIVTP with trainers, key clinic staff and the graduates of the program [8]. 
Informed consent will be collected from all survey participants through the form of a consent question at the beginning of the survey. The first question of the survey, before any data collection begins, will describe all aspects of the structured survey, all risks and benefits, and all plans for dissemination. Participants will be made aware that all participation is voluntary, anonymous, and can be discontinued at any time. In addition, contact information for the primary investigator will be provided. If participants consent to participate in the study, they will be progressed to the next set of questions. If participants do not consent to participate in the study, they will be thanked for their time and removed from the survey website (Appendix D)

Written consent forms will be handed out to all stakeholders before participation in the focus group and symposium. Written consent forms will present all risks and benefits, plans for dissemination of research material, and reassurance that all information collected will not be connected to any identifiers and will be kept completely anonymous (Appendix D).

\section{Focus Group and Symposium}

We will begin our evaluation with a discussion among the current St. Damien Training Program on-site faculty with respect to topic content, program length, practical skills, and lecture modalities. We will hold a symposium to review online resources for SDH HIVTP and catalogue these resources for in-person discussion with the SDH stakeholders. This program improvement exercise will inform new practices such as online computer modules, electronic reference materials, and packaging for program expansion. An anonymous evaluation form will be handed out to all participants following this exercise to elicit feedback and inform next steps for research. All data that is collected or audio recorded will have no identifiers used. Information collected from this symposium will be used solely for data collection and evaluation purposes.

\section{Interview/Survey Instruments}

Through an updated registry of participants we will distribute a translated program survey to each of the graduates by email (Appendix A). In addition, we will also ask for continued participation in the project including involvement in the focus group, and willingness to answer follow-up e-mails. After two unsuccessful e-mail attempts, phone contact will be initiated by a native speaker to administer the survey verbally. If an individual can't be reached through the contact information listed after program completion, investigators in conjunction with Haitian staff will reach out to hospitals, clinics, etc. where they were last known to have worked.

Content of the structured survey will be organized into domains: questions regarding total length of the program (i.e. impact on time away from clinical responsibilities outside of $\mathrm{SDH})$, topic content of the program and extent of lecture coverage (i.e. PMTCT, TB, malnutrition, etc.), lecture modalities (i.e. computer based vs. in-person), practical skills (i.e. pill counting, adherence counseling), and current engagement in pediatric HIV care.
The structured survey will be administered using DatStat Illume software, in a web-based self-administered format. The survey will be authenticated, meaning that individuals can only participate in the survey if they have a username and password. All program participants who were able to be contacted will receive a username and password at the time of survey distribution. Data collection through the DatStat Illume software will be completely anonymous and participants will only be tied back to their unique identifier number (which will be used as their username). Usernames will solely be used as a method of checking off who has completed the survey. We will hold one master list copy, stored in the HIVTP headquarters, and destroyed upon survey completion. All data will be stored in the DatStat Illume Web Console, which can only be accessed by the primary investigator. Once the online survey has been unpublished, so that nobody else can take the survey, data will be downloaded into a Microsoft Excel spreadsheet and stored on a computer at the HIVTP headquarters which requires a username and password to be accessed.

We will collect all pre--and post-test scores for evaluation of trainee performance. These scores will next be measured as a marker for continued engagement in HIV care. We will use a chi square test to measure the effect of program performance on continued engagement in HIV care.

Finally, to help improve the primary program outcome of training HIV providers, we will identify commonly encountered barriers and solutions in the primary-care setting. These will be identified through the structured survey and focus groups held with key informants of the program (program leaders, program graduates, social workers, psychologists, and other program stakeholders).

\section{Analysis}

Our analysis will include descriptive statistics of the program and quantitative and qualitative assessment of the cross-sectional survey and focus groups.

Quantitative analysis will be performed for the evaluation of the program curriculum with respect to topic content, program length, practical skills, and lecture modalities. Following the data collection through structured participant survey distributed to both trainees and trainers, data will be entered into STATA and all survey questions will be created as variables for data analysis.

In addition, new variables will be created for program performance through a compilation of pre- and post-test scores, as well as level of HIV care engagement following program completion. Statistical methods such as measures of association (odds ratio) will be used to look for correlation between amount of performance improvement between pre and post test and level of HIV care engagement following program completion.

Examination of clinical impact of the training program on children living with HIV in Haiti will be done through a descriptive analysis of level of clinical HIV activity. Variables 
for percentage of program participants providing pediatric HIV care, percentage of patients seen in their clinic with HIV, and what percentage of HIV patients seen in clinic is on ARV will be analyzed in order to determine the overall clinical impact of the program. In addition, level of HIV care prior to and following program completion will be measured and assessed in order to determine an increase or decrease in pediatric HIV care as a result of the training program.

This analysis will use the same data collected for the engagement in HIV care variable used previously and will measure the impact on pediatric HIV care in Haiti that has taken place due to the St. Damien training program. Level of HIV care prior to program participation and following program participation will be measured through the participant survey in order to determine how large the scaleup of the program was and to define the overall scope of the training program.

All pre- and post- test scores have already been collected and will be entered into STATA statistical program for analysis. Individual program performance will be determined through the scores and made into a categorical variable organized as "High," "Average," and "Low" performance. Level of HIV care engagement following program completion will be determined from the structured survey distributed to participants that will indicate whether or not they continue to see HIV patients, what percentage of their patients are pediatric HIV cases, and what percentage of their patients are on ARV. A categorical variable for continued engagement in HIV care will be generated that combines the variables mentioned previous and will be used as an engagement score. The two variables will then be analyzed for measures of association.

Qualitative analysis will be performed as a result of structured interviews and focus groups, with the purpose of identifying what successes of the program were for providers, as well as what barriers there were to completing the program and continuing HIV care with success. The intention is for identification of programmatic strengths and weaknesses, including identification of solutions to commonly encountered barriers of providing HIV care in the primary-care setting will be performed through focus groups held with key informants of the program (program leaders, program graduates, social workers, psychologists, and other program stakeholders).

\section{Risks and Benefits}

There are no potential risks for this study, as all information collected from surveys will be kept anonymous and will not be traced back to research participants. All qualitative information collected from focus groups will be kept anonymous and will not be traced back to individuals. Benefits include improving upon a successful and unique training program for physicians in the field of pediatric HIV and potentially providing a framework for other programs to develop in the future. See Appendix B for information on informed consent.

\section{Plan for Dissemination}

All data and research collected will be done in support of completion of my Brown University MPH thesis. Data results and analysis on the overall success and sustainability of the Saint Damien medical training program for HIV will be written up for publication. Among completion of program evaluation in August 2014, a comprehensive report on results and suggestions for future program operation will be presented to the directors of medical training for pediatric HIV at Saint Damien's Hospital. Results will also be shared with all participants and graduates of the Saint Damien training program in order to provide access to suggestions for best practices and strategies moving forward.

Data from the survey, focus group, and program outcomes will be compiled into a comprehensive report of the SDH HIVTP to help inform future practice $[9,10]$. Through this programmatic evaluation, our data will help inform our Haitian colleagues in developing a stronger framework for a more efficient HIVTP that could be scaled-up to meet the needs of Haiti.

\section{Detailed Budget}

Travel

- Airfare, round trip -- $\$ 600=\$ 600$

- Accommodations -- $\$ 26 /$ day $\times 56=\$ 1,456$

- Accommodations include 1 roundtrip travel to airport upon arrival, 1 meal per day, and internet access

- Travel to Logan International Airport, Boston

- Round trip train -- $\$ 30 \times 2=\$ 60$

- Planned chaperone site visits with NPH staff and security: $\$ 50 /$ trip for gas and driver $x \mathbf{4}=\$ \mathbf{2 0 0}$

\section{Communication}

- In country phone -- $\$ 40$ for phone, $\$ 50$ for 300 minute SIM card -- $\$ 90$

- Skype/Face Time credits -- $\mathbf{2 0}$

- Postage -- $\$ \mathbf{1 5}$

- Translators/Guides -- $\$ \mathbf{0 . 1 0}$ per word, $\mathbf{7 5 0 - 1 0 0}$ words $=\$ 100$

- $\$ 25 /$ day for 2 translators for focus groups $\times 2$ days $=\$ 50$

Other

- Sanford HIV Care Handbook for focus group participants: $\$ 35 \times 15=\$ 525$

- Photo copies of forms and surveys: In kind from Pediatric Department (\$200)

Other Support for the Project

Brown Graduate School International Travel Fund Grant

Awarded as $\$ 600$ to be used solely towards airfare

Appendix E: Focus Group Script and Questions

Focus Group Script - English 
1. Send out invitations to all focus group participants

2. The day before the focus group, send out a reminder to all participants

3. Prepare focus group guide

What we want to do: The Saint Damien HIV Training program has been preparing physicians for pediatric HIV care since 2008. We are interested in identifying the successes and barriers to the program since that time, so that the program can continue to improve and expand.

How the participants can help us achieve our goal: We would like your feedback on a number of different aspects of the training program. Your help is very valuable, because you can tell us what kinds of things are important to the training program and its success. As facilitators of the training program, you have the first-hand experience to what has and has not worked in the past.

\section{Means to record the session}

We will record the discussion in print for post-analysis. We will also audio record the discussion, should anything happen to the recorded notes. All recorded information is confidential and anonymous and will be used only for the purpose of this research project.

\section{Explain that the participants have the right to withdraw from the session at any time.}

You have the right to withdraw from the session at any time, should you no longer wish to participate. Choosing not to participate will have no bearing on your position in the training program.

\section{Introduce and ask the participants to sign the consent form}

I will now distribute a consent form for participation in today's focus group. Please take a couple minutes now to read over the form. If you have any questions before signing, feel free to ask me now. Once you have signed the form, please return them to me so that we may start the discussion.

\section{Discussion}

Now, we will move into the discussion portion of this focus group. I ask that only one person talks at a time. Remember that what is shared in the room stays in the room and all of your responses will be anonymous. There are no right or wrong answers to the questions, just ideas, experiences, and opinions, which are all valuable. If clarification is needed for any of the points, I can provide that to you. We expect the discussion to take about 1 hour.

1) How do you select program participants?

What is the application process?

Is selection competitive?

What are some of the challenges that you faced in the selection process?

2) How have you been measuring success of the program?
How do you use the program evaluations?

How useful do you think the evaluations were?

3) How do you keep in touch with past participants?

Do you send out yearly updates to all graduates?

Are there networking opportunities for graduates?

4) What do you think are the biggest successes of the training program?

Where have you seen the greatest improvement in the program?

What aspects of the program are the strongest?

5) What areas of the program that you think can improve?

Is there anything missing from the program due to resource constraints?

What aspects of the program do you think participants would want to see changed?

6) How do you feel about being recorded while teaching?

How would you feel about pre-recorded video lectures for the participants to watch on their own time?

7) How often has the program curriculum been revised?

What is the process for revisions for the curriculum?

Who decides how to revise the curriculum?

8) What opportunities do you think the program has for expanding to some online modules?

Do you see this as an opportunity to shorten the length of the program, so that participants spend less time away from their clinics?

\section{Closing the Session}

Have the note-taker summarize the findings and ask if there is anything that hasn't been discussed yet that the group would like to discuss.

Thank you all for your time and for your commitment to the training program. The focus group has now ended!

\section{Ways to Make Focus Group Run Smoothly}

- Only one person talks at a time

- Assure confidentiality: "What is shared in the room stays in the room."

- Hear everyone's ideas and opinions. There are no right or wrong answers to questions-just ideas, experiences, and opinions, which are all valuable

\section{References}

1. Annex to the Action Plan for the National Recovery and Development of Haiti. Haiti Earthquake Post-Disaster Needs Assessment (PDNA): Assessment of damage, losses, general and sectoral needs. 2010.

2. Convention on the Elimination of All Forms of Discrimination Against Women. Port-au-Prince, March 2008; 21.

3. George E, Noel F, Bois G, Cassagnol R, et al. Antiretroviral Therapy for HIV-1-Infected Children in Haiti. Oxford Journal JID. 2007: 195. 
4. HIV and AIDS Estimates. UNAIDS. 2012.

5. Fayorsey RN, Saito S, Carter RJ, et al. Decentralization of Pediatric HIV Care and Treatment in Five Sub-Saharan African Countries. JAIDS. 2013.

6. Persaud D, Gay H, Ziemniak C, Chen YH, Piatak M, et al. Absence of detectable HIV-1 viremia after treatment cessation in an infant. $N$ Engl J Med. 2013; 369(19): 1828-35.

7. Kimmel $A D$, Charles $M$, Deschamps $M M$, et al. Lives saved by expanding HIV treatment availability in resource-limited settings: the example of Haiti. J Acquir Immune DeficSyndr. 2013; 63(2): 40-8. doi: 10.1097/ QAl.0b013e3182918875
8. WHO. Global health observatory data repository, country-specific statistics, Haiti.

9. Abrams EJ, Simonds, RJ, Modi S, Rivadeneira E, Vaz P, Kankasa C, Tindyebwa D, Phelps R, Bowsky S, Teasdale C, Koumans E, Ruff AJ. PEPFAR Scale-up of Pediatric HIV Services: Innovations, Achievements, and Challenges. JAIDS. 2012.

10. Centers for Disease Control and Prevention. Guide to implementation of services for early infant diagnosis in resource-limited settings. 2009. 